\title{
Esophagitis resulting from treatment with crizotinib for anaplastic lymphoma kinase rearrangement-positive lung adenocarcinoma: A case report
}

\author{
OSAMU TAKAKUWA, TETSUYA OGURI, MIDORI YOKOYAMA, HISATOSHI HIJIKATA, \\ TAKEHIRO UEMURA, HIROTSUGU OHKUBO, KEN MAENO and AKIO NIIMI
} Department of Medical Oncology and Immunology, Nagoya City University, Graduate School of Medical Sciences,
Nagoya, Aichi 467-8601, Japan

Received April 24,2013; Accepted September 9, 2013

DOI: $10.3892 / \mathrm{mco} .2013 .188$

\begin{abstract}
Non-small-cell lung cancer (NSCLC) is the most commonly diagnosed type of cancer and is a leading cause of cancer-related mortality worldwide. Treatment is currently focused on individualization according to the molecular profile of the disease. Here we present the case of a 41-year-old patient who presented with multiple pulmonary nodules, a left pleural effusion and an ovarian tumor. Adenocarcinoma of the lung was diagnosed from pathological examination of the pleural effusion and the surgically resected ovarian tumor, and chemotherapy was initiated. Relapse was experienced following third-line chemotherapy with pemetrexed and anaplastic lymphoma kinase (ALK)-positive adenocarcinoma was diagnosed using a specimen from the resected ovarian tumor. Subsequently, crizotinib therapy was initiated. Eight days later the patient developed severe nausea and vomiting and esophagitis was diagnosed by gastrointestinal endoscopic examination. Following the interruption of crizotinib treatment by treatment with a proton pump inhibitor (PPI), crizotinib treatment was re-initiated and was effective for a minimum of 6 months. Clinicians should be aware that treatment with crizotinib may result in severe esophagitis.
\end{abstract}

\section{Introduction}

Non-small-cell lung cancer (NSCLC) is the most commonly diagnosed type of cancer and is a leading cause of cancer-related mortality worldwide (1). Currently, strategies aiming to maximize treatment benefit for NSCLC are centered on individualized treatments based on the molecular profile of

Correspondence to: Dr Tetsuya Oguri, Department of Medical Oncology and Immunology, Nagoya City University, Graduate School of Medical Sciences, 1 Kawasumi, Mizuho-cho, Mizuho-ku, Nagoya, Aichi 467-8601, Japan

E-mail: t-oguri@med.nagoya-cu.ac.jp

Key words: anaplastic lymphoma kinase, crizotinib, non-small-cell lung cancer, pemetrexed the disease. The identification of mutations of the epidermal growth factor receptor $(E G F R)$ gene and development of its tyrosine kinase inhibitors have signifficantly affected the potency of the response to NSCLC treatment and has led to additional oncogenic drivers being aggressively investigated.

Anaplastic lymphoma kinase (ALK) rearrangements in NSCLC were first described in 2007 (2). Several ALK inhibitors have been introduced in clinical trials or are currently under preclinical development. Among these, crizotinib was shown to possess marked therapeutic efficacy (3) and was approved by the US Food and Drug Administration (FDA) in 2011, shortly after its development.

Crizotinib is used worldwide for the treatment of ALK-positive NSCLC; however, there is increasing accumulation of data with regard to its potential adverse events, which may be severe. Here we present the case report of a patient with ALK-positive NSCLC and severe esophagitis caused by crizotinib treatment.

\section{Case report}

A 41-year-old woman was admitted to our hospital with a left pleural effusion. Adenocarcinoma cells were detected by cytological examination of the pleural effusion. Whole-body computed tomography $(\mathrm{CT})$ revealed multiple nodular shadows in the left pulmonary field, a left pleural effusion and a multilocular tumor in the right ovary (Fig. 1A and B). The ovarian tumor was surgically resected and immunohistochemistry (IHC) revealed characteristics of a primary lung adenocarcinoma. Active EGFR mutations were not identified. This study was approved by the ethics committee of Nagoya City University (Nagoya, Japan). The patient provided their consent for their participation in the study.

Following pleurodesis with OK-432, the patient was treated with one course of carboplatin [area under the curve (AUC) $=6$ on day 1, every 3-4 weeks] and paclitaxel $\left(200 \mathrm{mg} / \mathrm{m}^{2}\right.$ on day 1 , every 3-4 weeks) as first-line chemotherapy and 12 courses of docetaxel $\left(60 \mathrm{mg} / \mathrm{m}^{2}\right.$ on day 1 , every 3-4 weeks) as second-line chemotherapy. Following a relapse with docetaxel (Fig. 1C), pemetrexed $\left(500 \mathrm{mg} / \mathrm{m}^{2}\right.$ on day 1 , every $3-4$ weeks) was administered as third-line chemotherapy, which resulted in a 
A

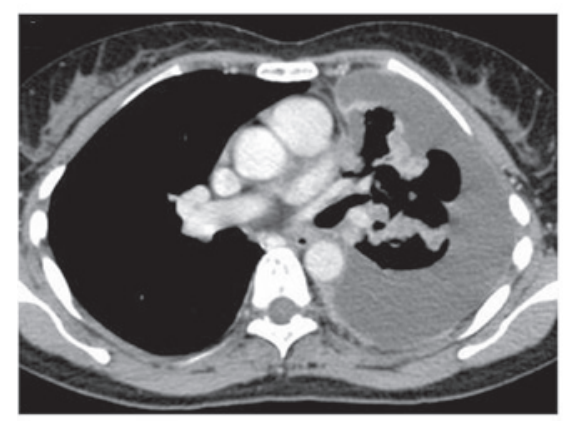

C

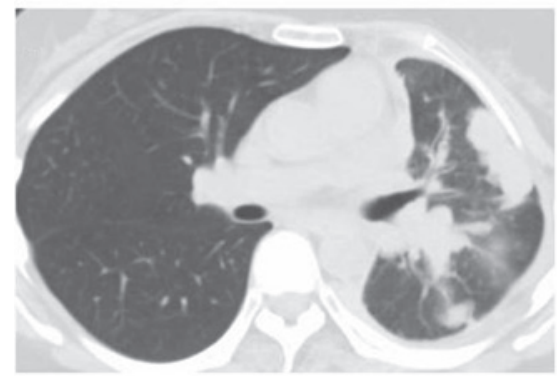

B

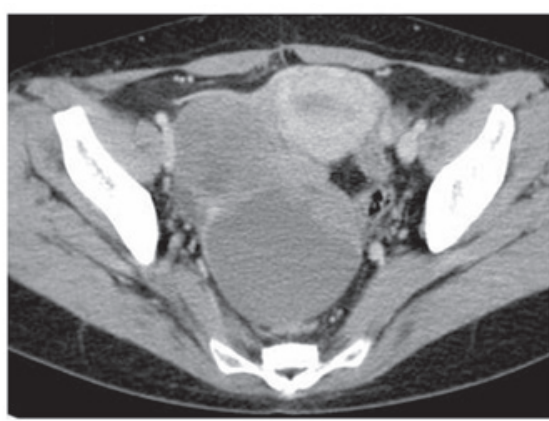

D

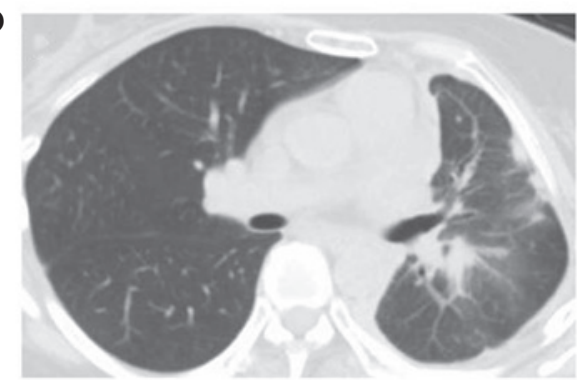

Figure 1. (A) Chest computed tomography (CT) at diagnosis revealed multiple pulmonary nodules in the left upper lobe and a pleural effusion. (B) Pelvic CT at diagnosis revealed a multilocular tumor in the right ovary. (C) Chest $\mathrm{CT}$ at the start of pemetrexed treatment revealed pulmonary tumors in the left upper lobe with enlargement of the hilar lymph nodes. (D) At the start of crizotinib treatment, the lung tumors and hilar lymph nodes remained reduced in size.

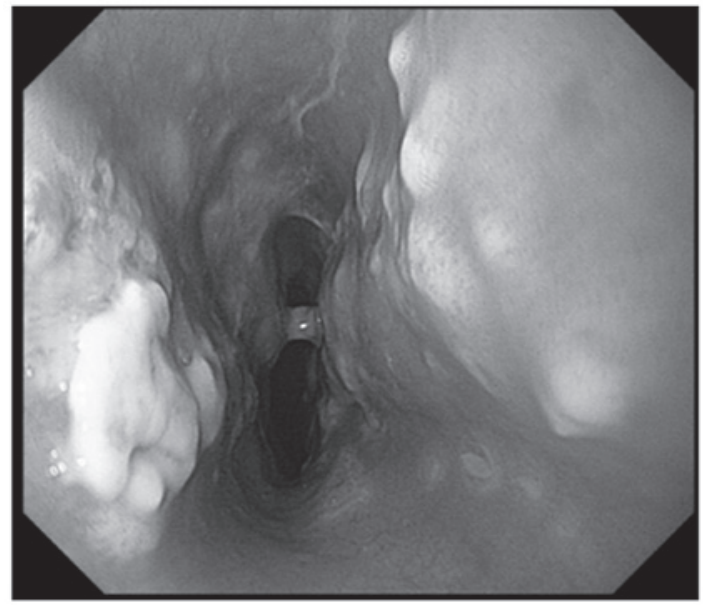

Figure 2. Gastrointestinal endoscopic examination 10 days after the initiation of crizotinib treatment revealed multiple erosive lesions with a white coating in the middle thoracic portion of the esophagus.

partial response. This treatment was continued for 36 months (34 cycles) (Fig. 1D). However, the patient relapsed with bone metastasis of the 10th thoracic vertebra and elevation of serum carcinoembryonic antigen (CEA) levels (from 3.5 to $8.4 \mathrm{ng} / \mathrm{ml}$ ). ALK expression and translocation were detected via IHC and fluorescence in situ hybridization (FISH) analysis of the surgically resected ovarian tumor. Therefore, crizotinib treatment $(500 \mathrm{mg} /$ day) was initiated as fourth-line treatment following the administration of 39 Gy of radiotherapy to the metastatic lesion in the thoracic vertebra.

Two days after the initiation of crizotinib, the patient developed nausea and vomiting. Eight days later, the patient was unable to eat or drink. Gastrointestinal endoscopic examination 10 days after the initiation of crizotinib revealed severe esophagitis in the middle thoracic portion (Fig. 2), estimated to be of grade 3 severity. Crizotinib treatment was discontinued and intravenous nutrition and proton pump inhibitors (PPI) were administered, following which the symptoms subsided and crizotinib was re-initiated one week later at a dose of $400 \mathrm{mg} / \mathrm{day}$, along with ongoing PPI therapy. There was no recurrence of the esophageal symptoms. Two months after the initiation of crizotinib treatment the CEA levels decreased to $3.9 \mathrm{ng} / \mathrm{ml}$. Four months later the CEA levels were within the normal range and a $\mathrm{CT}$ examination revealed no evidence of recurrence.

\section{Discussion}

In phase I and II trials, crizotinib was shown to be highly effective in patients with advanced ALK-positive NSCLC, with overall response rates 50-60\% (3). In the phase I trial, the median progression-free survival (PFS) was 10 months (4), which was similar to that achieved with EGFR inhibitors in advanced EGFR-mutation NSCLC. Crizotinib is currently recognized to have important implications for the management of patients with ALK-positive NSCLC. In the present case, crizotinib was used as a fourth-line therapy and a therapeutic effect was achieved. Shaw et al (5) reported that ALK-positive NSCLC patients exhibited a longer overall survival following crizotinib administration as second- or third-line therapy in a retrospective case-matched analysis. More recently, crizotinib as second-line treatment for patients with ALK-positive NSCLC achieved longer PFS compared with chemotherapy using docetaxel or pemetrexed in phase III trials (6). Therefore, it may be suggested that crizotinib should be used for either course of treatment in patients with ALK-positive NSCLC.

With regard to the adverse effects, crizotinib was demonstrated to be tolerable $(3,4)$. The most frequently occurring treatment-related adverse events are visual disturbances, 
gastrointestinal events (nausea, diarrhea, vomiting and constipation) and peripheral edema $(3,4)$. However, these events tend to be mild and grade $3 / 4$ adverse events are rare $(3,4)$. In the present case, grade 3 esophagitis resulted from treatment with crizotinib. Esophagitis has not been reported as a major crizotinib-related adverse event in previous studies, although three cases of esophagitis (two cases of grade 1 and one of grade 2) were reported among 255 patients in phase I and II trials (7). Furthermore, at the time of writing, in post-marketing surveillance in Japan (8), three cases of esophageal ulcers and six of esophagitis caused by crizotinib were reported; among these nine cases, three were severe. Additionally, the same study listed several adverse events which could be associated with the esophagus, such as heartburn and reflux esophagitis (8). Gastrointestinal endoscopy is usually required for the diagnosis of esophagitis, which supports the hypothesis that more cases of undocumented esophagitis may have occurred in patients undergoing crizotinib treatment compared with what was actually reported. In the present case, crizotinib treatment was re-initiated following a brief interruption and the initiation of PPI therapy. Esophagitis should be recognized as a potential adverse event of crizotinib treatment and, if diagnosed, early treatment with PPI may aid in the continuation of crizotinib treatment.

In the present case, ALK-positive cells were diagnosed using formalin-fixed, paraffin-embedded (FFPE) tissues that had been obtained five years previously. IHC and FISH are the primary methods for the detection of ALK-positive NSCLC (9) and are applicable to FFPE tissue specimens $(10,11)$. Therefore, this disease may be diagnosed using previously obtained samples. Since re-examination of biopsies is usually not feasible in advanced NSCLC patients, this is of clinical significance. Furthermore, in the present case, the FFPE tissue specimen used for determining ALK-positivity was obtained from a metastatic tumor of the ovary. However, it has been previously reported that there may be a difference in EGFR and KRAS gene status between primary and metastatic tumor samples of NSCLC (12). Further studies are required in order to evaluate the adequacy of pathological samples obtained from metastatic tumors for the diagnosis of ALK-positivity of NSCLC.

In this case, the patient exhibited an eminently prolonged response to pemetrexed, which preceded crizotinib treatment. Pemetrexed is a promising treatment for NSCLC with non-squamous cell histology, although this drug was recently reported to have a prominent activity in ALK-positive NSCLC. Among NSCLC patients treated with pemetrexed, the median PFS in ALK-positive patients was nine months, compared with four months in an EGFR, ALK and KRAS wild-type control group (13). Two initial hypotheses were considered to explain the 'super-sensitivity' of ALK-positive NSCLC to pemetrexed (14). Firstly, ALK positivity is associated with lower levels of thymidylate synthase, one of the targets of pemetrexed (15). Secondly, 5-aminoimidazole-4-carboxamide ribonucleotide formyltransferase/inosine monophosphate cyclohydrolase, a catalytic enzyme, may be a substrate for
ALK-mediated phosphorylation and a direct target for pemetrexed (13). It is possible that the efficacy of pemetrexed in the present case was associated with ALK positivity. Further studies are required in order to determine treatment strategies involving pemetrexed and crizotinib in patients with ALK-positive NSCLC.

In summary, the present case report describes a patient with ALK-positive lung adenocarcinoma who developed severe esophagitis following treatment with crizotinib. Clinicians should be aware that crizotinib therapy may result in severe esophagitis.

\section{References}

1. Janku F, Stewart DJ and Kurzrock R: Targeted therapy in non-small-cell lung cancer - is it becoming a reality? Nat Rey Clin Oncol 7: 401-414, 2010.

2. Soda M, Choi YL, Enomoto M, et al: Identification of the transforming EML4-ALK fusion gene in non-small-cell lung cancer. Nature 448: 561-566, 2007.

3. Kwak EL, Bang YJ, Camidge DR, et al: Anaplastic lymphoma kinase inhibition in non-small-cell lung cancer. N Engl J Med 363: 1693-1703, 2010.

4. Camidge DR, Bang YJ, Kwak EL, et al: Activity and safety of crizotinib in patients with ALK-positive non-small-cell lung cancer: updated results from a phase 1 study. Lancet Oncol 13: 1011-1019, 2012.

5. Shaw A, Yeap B, Solomon B, et al: Impact of crizotinib on survival in patients with advanced, ALK-positive NSCLC compared with historical controls. J Clin Oncol 29 (Suppl): abs. 7507, 2011

6. Shaw A, Kim D, Nakagawa K, et al: Phase III study of crizotinib versus pemetrexed or docetaxel chemotherapy in patients with advanced ALK-positive non-small cell lung cancer (NSCLC) (PROFILE 1007). Ann Oncol 23 (Suppl 10): abs. LBA1_PR 2012.

7. Pfizer: Crizotinib adverse drug reactions reported from the clinical trials (PROFILE 1001 study and PROFILE 1005 study).

8. Pfizer: Xalkori adverse drug reactions reported from the post-marketing surveillance in Japan.

9. Thunnissen E, Bubendorf L, Dietel M, et al: EML4-ALK testing in non-small cell carcinomas of the lung: a review with recommendations. Virchows Arch 461: 245-257, 2012.

10. Takeuchi K, Choi YL, Togashi Y, et al: F5B-ALK, a novel fusion oncokinase identified by an immunohistochemistry-based diagnostic system for ALK-positive lung cancer. Clin Cancer Res 15: 3143-3149, 2009.

11. Mino-Kenudson M, Chirieac LR, Law K, et al: A novel, highly sensitive antibody allows for the routine detection of ALK-rearranged lung adenocarcinomas by standard immunohistochemistry. Clin Cancer Res 16: 1561-1571, 2010.

12. Kalikaki A, Koutsopoulos A, Trypaki M, et al: Comparison of EGFR and K-RAS gene status between primary tumours and corresponding metastases in NSCLC. Br J Cancer 99: 923-929, 2008.

13. Camidge DR, Kono SA, Lu X, et al: Anaplastic lymphoma kinase gene rearrangements in non-small cell lung cancer are associated with prolonged progression-free survival on pemetrexed. J Thorac Oncol 6: 774-780, 2011.

14. Camidge DR and Doebele RC: Treating ALK-positive lung cancer - early successes and future challenges. Nat Rev Clin Oncol 9: 268-277, 2012.

15. Lee JO, Kim TM, Lee SH, et al: Anaplastic lymphoma kinase translocation: a predictive biomarker of pemetrexed in patients with non-small cell lung cancer. J Thorac Oncol 6: 1474-1480, 2011. 